\title{
ELECTROCARDIOGRAPHIC CHANGES DURING POSITIVE ACCELERATION
}

\author{
BY \\ M. K. BROWNE AND J. T. FITZSIMONS
}

From the R.A.F. Institute of Aviation Medicine, Farnborough, and the Physiology Laboratory, Cambridge

Received February 14, 1958

The effects of accelerative forces on man are of considerable importance in high performance aircraft. Should manned space travel become possible then one of the many medical problems will be the reaction of man to varying gravitational forces. To study the effects of positive acceleration (or g) a man-carrying centrifuge has recently been installed at the Institute of Aviation Medicine, Farnborough. By means of this machine, human beings can be exposed to controlled accelerative forces and their physiological reactions analysed in the laboratory.

In the early stages of operating the human centrifuge it was decided that objective methods of monitoring subjects' reactions to $\mathrm{g}$ were required, partly as a safety precaution and partly to provide basic data for future experiments. Electrocardiography was investigated because during $g$ the heart is subject to considerable stress, and because of the following advantages.

1. A continuous record of heart rate could be obtained.

2. It was known that $g$ influenced the position of the heart and in the absence of $X$-ray facilities it was thought that the electrical position of the heart would provide valuable information.

3. The technique was simple enough to be used as a routine.

This paper comprises the results of this investigation.

\section{METHODS}

The electrocardiographic leads used were the standard bipolar leads I, II, and III, the augmented unipolar limb leads and the unipolar chest leads, placed in accordance with the recommendations of the American Heart Association and the British Cardiac Society. The Wilson indifferent electrode was used (Wilson et al., 1934). To obviate muscle artefacts, extremely difficult to do under $\mathrm{g}$, and to allow free use of the hands, the two wrist electrodes were replaced by disc electrodes on the acromial processes. The potentials measured at the shoulders are very little different from those found at the wrists. In both cases the potentials are being recorded essentially from the root of the upper limb and any difference in length of conducting medium is of little significance when compared with the skin resistance.

Small non-polarizable silver electrodes were used attached to the skin by nobecutanec (Browne, 1957). The signals from the subject were led through a distribution box, enabling any number of lead positions to be recorded simultaneously. After suitable amplification they were displayed on a 12-channel Ediswan pen recorder. When higher quality records were required a high speed galvanometer was used. It was found necessary to use a 15 c.p.s. filter to reduce muscle artefacts under $\mathrm{g}$; this causes some reduction in the amplitude of the QRS complex due to high-frequency cut-out.

Respiration was recorded by means of a small thermocouple placed in one nostril. Changes in thermal e.m.f. due to passage of inspired and expired air were recorded as a low-frequency wave 
on the pen recorder. Subjects were exposed to forces of $3,3 \cdot 5$, and $4 \mathrm{~g}$, the force acting from head to feet (this is defined as positive acceleration). Unless otherwise stated all runs lasted for 15 seconds; this excludes the times taken to reach peak $g$ and to slow down at the end of the run. Two groups of subjects participated: $(a)$ experienced subjects who are members of the Institute of Aviation Medicine Staff, and $(b)$ healthy subjects with no previous centrifuge experience.

\section{RESULTS}

In this study the results of 366 centrifuge runs on 53 subjects have been analysed for changes in pulse rate, alterations in cardiographic pattern, and variation in the mean instantaneous electrical axis.

(i) The Pulse Rate. During positive acceleration the heart rate increases. In Table I, the resting pulse rate, the maximum rate reached, and the time from the start of the run to reach maximum rate are given for experienced and inexperienced subjects at different levels of $\mathrm{g}$. Peak $\mathrm{g}$ occurs in all cases 9 seconds after the start of the run.

TABLE I

Variations of Pulse Rate in Experienced and IneXperienced Subjects in Human Centrifuge

\begin{tabular}{|c|c|c|c|c|c|c|c|c|c|c|}
\hline \multirow[b]{2}{*}{$\mathbf{g}$} & \multicolumn{5}{|c|}{ Experienced } & \multicolumn{5}{|c|}{ Inexperienced } \\
\hline & $\begin{array}{l}\text { No. of } \\
\text { subjects }\end{array}$ & $\begin{array}{l}\text { Resting } \\
\text { pulse } \\
\text { rate }\end{array}$ & $\begin{array}{l}\text { Maximum } \\
\text { pulse } \\
\text { rate }\end{array}$ & $\begin{array}{l}\text { Per cent } \\
\text { increase }\end{array}$ & $\begin{array}{c}\text { Time to } \\
\text { maxi- } \\
\text { mum } \\
\text { sec. }\end{array}$ & $\begin{array}{c}\text { No. of } \\
\text { subjects }\end{array}$ & $\begin{array}{l}\text { Resting } \\
\text { pulse } \\
\text { rate }\end{array}$ & $\begin{array}{c}\text { Maximum } \\
\text { pulse } \\
\text { rate }\end{array}$ & $\begin{array}{l}\text { Per cent } \\
\text { increase }\end{array}$ & $\begin{array}{l}\text { Time to } \\
\text { maximum } \\
\text { sec. }\end{array}$ \\
\hline $\begin{array}{l}3 \cdot 0 \\
3 \cdot 5 \\
4 \cdot 0\end{array}$ & $\begin{array}{r}12 \\
11 \\
3\end{array}$ & $\begin{array}{l}76 \cdot 5 \\
85 \\
77\end{array}$ & $\begin{array}{r}97 \\
121 \\
114\end{array}$ & $\begin{array}{l}23 \cdot 5 \\
42 \cdot 3 \\
48\end{array}$ & $\begin{array}{l}16 \cdot 6 \\
14 \cdot 3 \\
16 \cdot 1\end{array}$ & $\begin{array}{l}20 \\
20 \\
12\end{array}$ & $\begin{array}{l}82 \cdot 3 \\
86 \\
83 \cdot 4\end{array}$ & $\begin{array}{l}120 \cdot 3 \\
127 \cdot 3 \\
129 \cdot 9\end{array}$ & $\begin{array}{l}47 \cdot 4 \\
48 \cdot 5 \\
55 \cdot 8\end{array}$ & $\begin{array}{l}12 \cdot 1 \\
13 \cdot 9 \\
14 \cdot 6\end{array}$ \\
\hline
\end{tabular}

At $3 \mathrm{~g}$ the difference in maximum pulse rate between experienced and inexperienced subjects is significant at the 1 per cent level, but at 3.5 and $4 \mathrm{~g}$ there is no significant difference. Likewise the time to maximum pulse rate shows a significant difference at the $0 \cdot 2$ per cent level at $3 \mathrm{~g}$ between experienced and inexperienced subjects but no difference at higher $\mathrm{g}$.

A number of runs were carried out wearing a standard anti-g suit which exerts counter pressure over the legs and abdomen during positive acceleration; this prevents pooling of blood in the lower part of the body and descent of the diaphragm. The results obtained show that wearing an anti-g suit makes little difference to the maximum pulse rates reached, but shortens the time taken to reach maximum pulse rate by 18 per cent at $3.5 \mathrm{~g}$ and 21 per cent at $4.0 \mathrm{~g}$

The $\mathrm{P}-\mathrm{R}$ interval represents the time taken by the stimulus to spread from the sinu-atrial node to ventricles and varies inversely as the heart rate. The $P-R$ interval has been measured in some runs and the results are given in Table II. Each result is the mean of five observations.

TABLE II

EFFECT OF g ON P-R INTERVAL

\begin{tabular}{|c|c|c|c|c|c|c|c|}
\hline \multirow[b]{2}{*}{$\mathbf{g}$} & \multirow{2}{*}{$\begin{array}{l}\text { Static } \\
\text { sec. }\end{array}$} & \multicolumn{6}{|c|}{ Stage of acceleration (time from start of acceleration) } \\
\hline & & $\begin{array}{c}0 \\
\text { sec. }\end{array}$ & $\begin{array}{r}2 \cdot 5 \\
\mathrm{sec} .\end{array}$ & $\begin{array}{r}7 \cdot 5 \\
\text { sec. }\end{array}$ & $\begin{array}{l}12 \cdot 5 \\
\mathrm{sec} .\end{array}$ & $\begin{array}{l}17 \cdot 5 \\
\mathrm{sec} .\end{array}$ & $\begin{array}{c}22 \cdot 5 \\
\mathrm{sec} .\end{array}$ \\
\hline $\begin{array}{l}3 \cdot 0 \\
3 \cdot 5 \\
4 \cdot 0 \\
4 \cdot 5 \\
5 \cdot 0\end{array}$ & $\begin{array}{l}0 \cdot 15 \\
0 \cdot 14 \\
0 \cdot 137 \\
0 \cdot 14 \\
0 \cdot 14\end{array}$ & $\begin{array}{l}0 \cdot 15 \\
0 \cdot 13 \\
0 \cdot 14 \\
0 \cdot 13 \\
0 \cdot 14\end{array}$ & $\begin{array}{l}0 \cdot 13 \\
0 \cdot 12 \\
0 \cdot 125 \\
0 \cdot 12 \\
0 \cdot 12\end{array}$ & $\begin{array}{l}0 \cdot 125 \\
0 \cdot 11 \\
0 \cdot 12 \\
0 \cdot 105 \\
0 \cdot 10\end{array}$ & $\begin{array}{l}0 \cdot 12 \\
0 \cdot 10 \\
0 \cdot 11 \\
0 \cdot 10 \\
0 \cdot 10\end{array}$ & $\begin{array}{l}0 \cdot 13 \\
0 \cdot 11 \\
0 \cdot 12 \\
0 \cdot 12 \\
0 \cdot 11\end{array}$ & $\begin{array}{l}0 \cdot 14 \\
0 \cdot 125 \\
0 \cdot 13 \\
0 \cdot 13 \\
0 \cdot 12\end{array}$ \\
\hline
\end{tabular}


Analysis of variance shows that the sample was a representative one of the population and that the change in $\mathrm{P}-\mathrm{R}$ interval during $\mathrm{g}$ was significant at the 0.1 per cent level as was also the progressive change in $\mathrm{P}-\mathrm{R}$ interval with higher $\mathrm{g}$ levels. It will be seen that the $\mathrm{P}-\mathrm{R}$ interval usually started to decrease as acceleration commenced, and at the end of the run started to return to normal while the acceleration was still being applied. This corresponds to the behaviour of the pulse, which reaches its maximum towards the middle of the run and then begins to fall again. Both changes can be related to the behaviour of the arterial blood pressure (Fig. 1). Throughout the series all $\mathrm{P}-\mathrm{R}$ intervals fell within the normal range of $0 \cdot 1-0 \cdot 2 \mathrm{sec}$. indicating that there was no abnormality of auriculo-ventricular conduction.

(a)

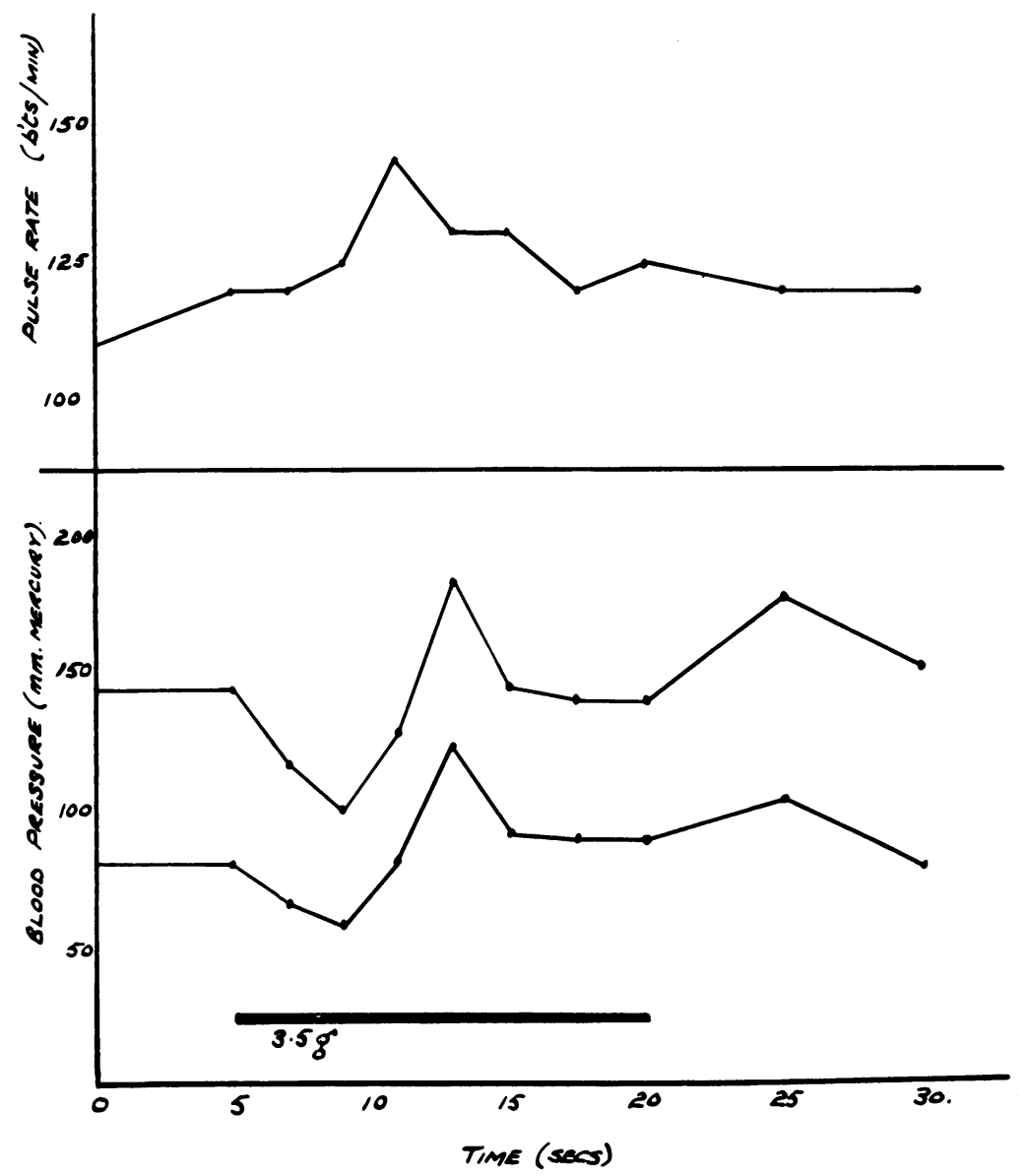

FIG. 1.-Graphs, showing simultaneous changes in $(a)$ pulse rate and $(b)$ arterial blood pressure at eye level during positive acceleration.

(ii) Form of the Electrocardiogram. No characteristic abnormalities were found in this investigation. Most runs were of short duration, but a number of runs lasting up to 5 minutes have been made; even in these no change other than that due to change in electrical axis was noted. On one occasion when the subject lost consciousness it was possible to get a record (Fig. 2). Unfortunately it is not of high quality owing to a certain amount of base line shift. This record, however, is the nearest approach to a left ventricular strain pattern we have obtained and as can be seen the evidence is dubious. It is possible that older subjects with diseased hearts may show more convincing changes. 

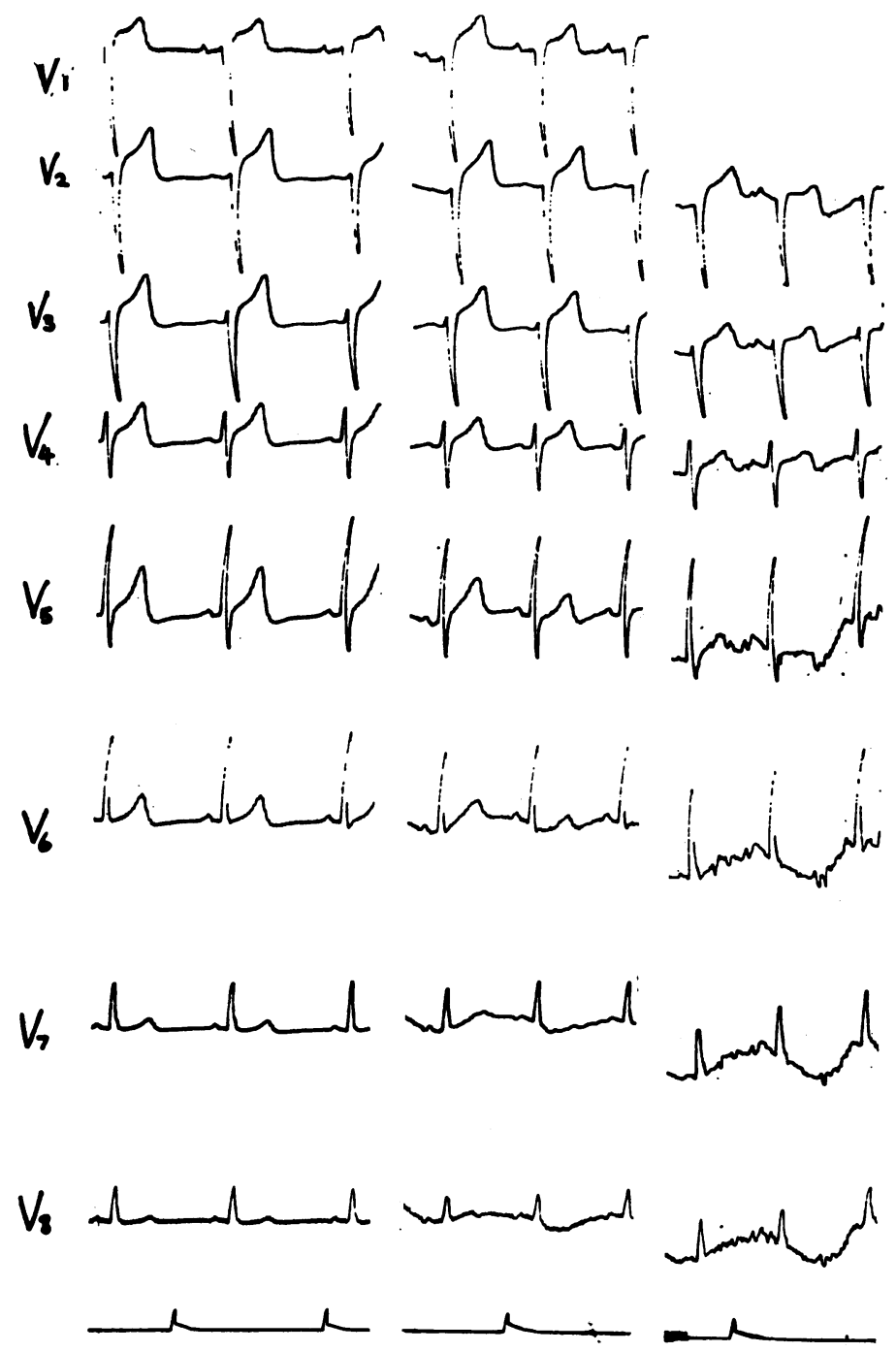

(a)

(b)

(c)

FIG. 2.-Multichannel cardiograms $(a)$ at rest, $(b)$ at $4.0 \mathrm{~g},(c)$ at $4.0 \mathrm{~g}$ during unconsciousness. All traces are at the same phase of respiration. When the subject loses consciousness there is a suppression of the $T$ waves.

(iii) The Electrical Axis. The mean instantaneous electrical axis was calculated by the method of Goldberger (1953). Each result was the mean of three observations, for it was rarely possible to obtain more than three full cycles of respiration owing to the short duration of the runs. However, in any one subject, figures for the same phase of respiration were usually identical. Furthermore, in three subjects, changes in axis observed by direct vector-cardiography (Browne and Fitzsimons, 1957) showed close agreement with the derived axis. An example of the changes found is shown graphically in Fig. 3. Five out of eight subjects (P. H., N. K., R. B., P. G., J. T. F.) had vertical hearts as judged from the electrical axis (i.e. more than $+45^{\circ}$ in the frontal plane) and two (M. S., M. K. B.) had horizontal hearts. One subject (F. Z.) was borderline, $39^{\circ}-45^{\circ}$.

Respiration has a marked effect on the electrical axis in most cases; this may be deduced from the effect on the cardiogram (Fig. 4). In the frontal plane the heart becomes more vertical with inspiration. In the sagittal plane the heart also becomes more vertical though the movement is 


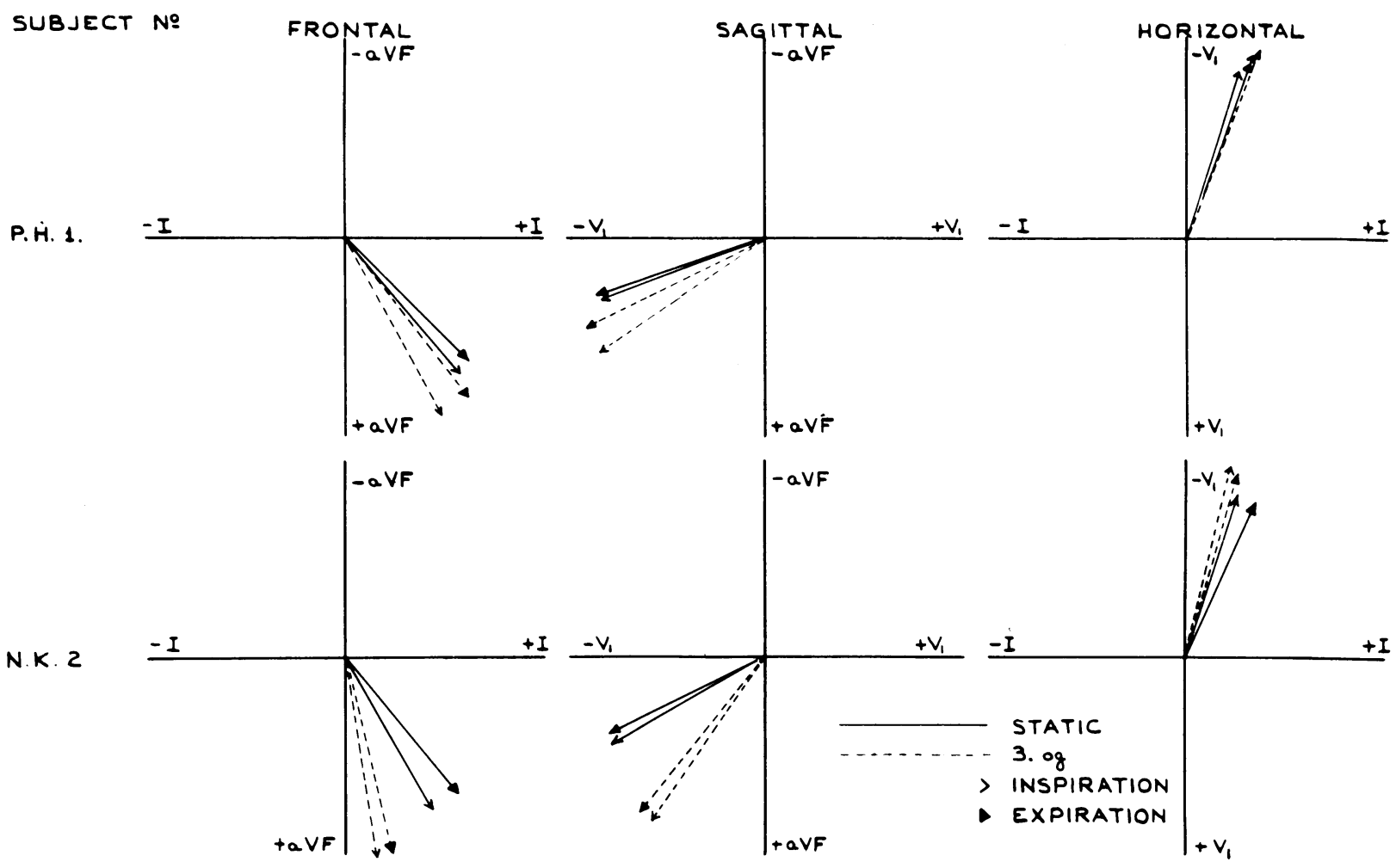

Fig. 3.-Two examples of the change in the manifest electrical axis of the heart during respiration and during the application of $3 \mathrm{~g}$.

rather less. In two subjects, R. B. and M. S., this axis tended to become more horizontal by a negligible amount. In the horizontal plane viewed from below the changes were small, two subjects showing clockwise rotation (J. T. P., P. G.), five anti-clockwise rotation, and one subject showing no movement (R. B.).

When subjected to positive acceleration of $4 \mathrm{~g}$, the respiratory changes are often even greater than at rest at $1 \mathrm{~g}$ (Fig. 5). However, there is an overall shift in the mean axis. The frontal and sagittal axes become more vertical and the horizontal axis rotates in an anti-clockwise direction in subjects with vertical hearts at $1 \mathrm{~g}$. In the two subjects with horizontal hearts at rest (M. S. and M. K. B.) the changes in all three planes are the opposite of those occurring in subjects with vertical hearts.

The changes in mean axis between $1 \mathrm{~g}$ and $3 \mathrm{~g}$ are summarized in Table III in which positive signs denote clockwise rotation.

TABLE III

Change in Mean Electrical Axis of the Heart at 3 G

\begin{tabular}{l|c|c|c}
\hline \multicolumn{1}{c|}{ Subject } & Frontal & Sagittal & Horizontal \\
\cline { 1 - 1 } P. H. & +10 & -11 & -2 \\
J. F. & +11 & -1 & +9 \\
N. K. & +24 & -25 & +6 \\
P. G. & +3 & -6 & +13 \\
F. Z. & +2.5 & -5 & +2 \\
R. B. & +9.5 & 0 & +8 \\
M. S. & -10 & +6.5 & -1 \\
M. K. B. & -4 & +1 & -6 \\
\hline
\end{tabular}




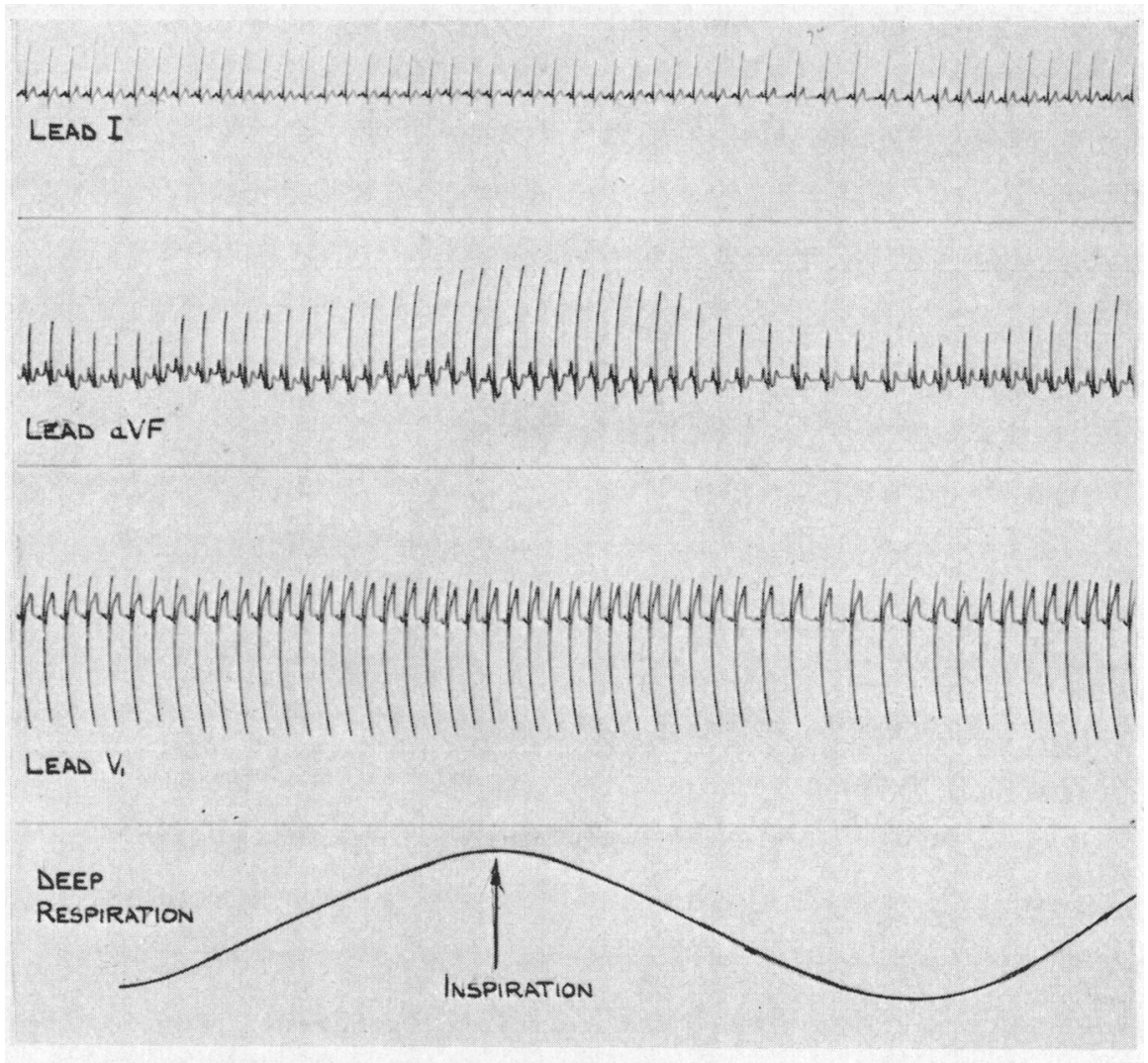

FIG. 4.-The effect of deep respiration on the electrocardiogram at rest. Note particularly the effect on amplitude of QRS and on the T waves.

To try to relate the changes in electrical axis to alterations in the anatomical position of the heart, chest X-ray pictures of three subjects were taken in full inspiration and full expiration. The long axis of the heart derived from these radiographs is compared with the frontal manifest electrical axis in Table IV.

TABLE IV

Comparison of Changes in Anatomical and Electrical Axis During Deep INSPIRATION AND EXPIRATION

\begin{tabular}{l|c|c|c|c|c|c}
\hline \multirow{2}{*}{ Subject } & \multicolumn{3}{|c|}{ Anatomical Axis } & \multicolumn{3}{c}{ Electrical Axis } \\
\cline { 2 - 7 } & Inspiration & Expiration & Range & Inspiration & Expiration & Range \\
\hline J. T. F. & +51 & +33 & +18 & +84 & +42 & +42 \\
P. G. & $+58 \cdot 5$ & +40 & $+18 \cdot 5$ & +83 & +78 & +5 \\
M. K. B. & $+39 \cdot 7$ & +36 & $+3 \cdot 7$ & +67 & +18 & +49 \\
\hline
\end{tabular}

\section{Discussion}

The causes of the increase in pulse rate during exposure to positive $\mathrm{g}$ are not completely understood. An important factor is the fall in blood pressure in the carotid sinus, and it may be seen that this fall precedes the maximum increase in heart rate (Fig. 1). Another possible cause, however, 


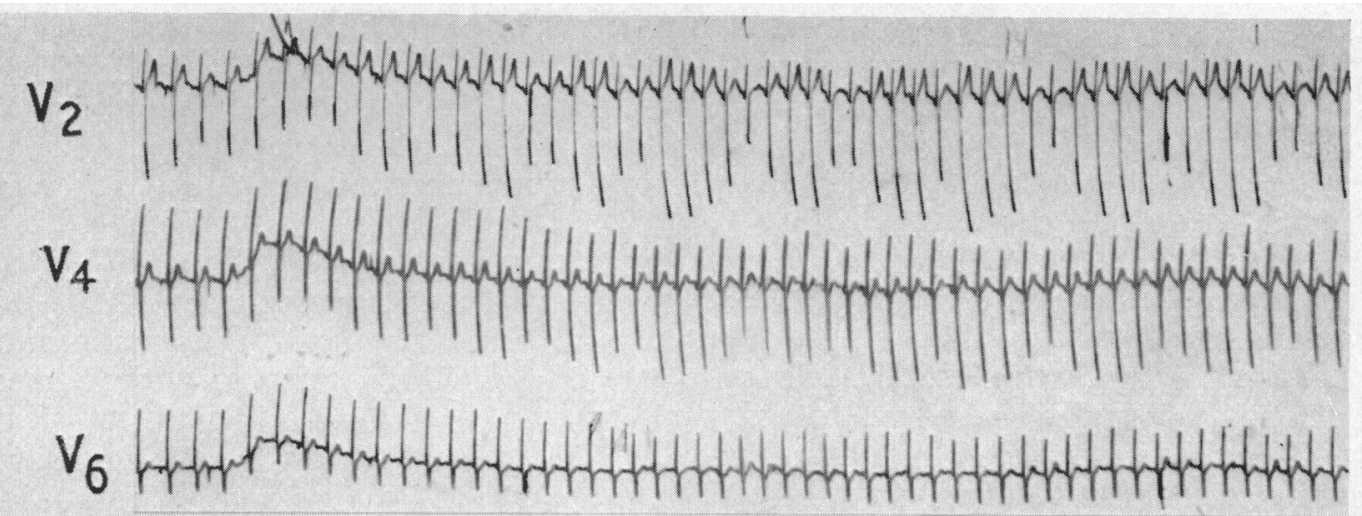

ACCELEROMETER

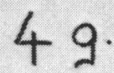

TIME is SECS.

FIG. 5.-The electrocardiogram during positive acceleration. This shows the increased effect of respiration under $\mathrm{g}$ and the enhanced $\mathrm{T}$ wave modulation which accompanies it.

is the adrenal medullary response to stress. At $3 \mathrm{~g}$ there is a significant difference in maximum pulse rate reached between experienced and inexperienced subjects. If fall in blood pressure were the only factor causing an increase in pulse rate, there would be no difference between the two groups of subjects. However, the fact that at $3 \mathrm{~g}$ inexperienced subjects show an earlier and greater pulse rate response suggests that in these subjects other mechanisms play a more important part. Two factors suggest themselves, first an involuntary tightening of the abdominal and leg musculature which tends to maintain venous return to the heart, and secondly release of adrenal medullary hormones resulting from apprehension at being subjected to an unusual manœuvre. In support of the first is the more rapid pulse response when wearing an anti-g suit. The anti-g suit maintains venous return in much the same way as involuntary muscular effort and presumably excites a Bainbridge reflex. In support of the second factor, von Euler and Lundberg (1954) have found an increased urinary output of catechol amines in inexperienced personnel when flying. At higher $g$ levels, where a maximum response is required on hæmodynamic grounds, the difference in maximum pulse rates tends to disappear, though inexperienced subjects still tend to reach maximum values sooner, suggesting that apprehension is still having a significant effect.

Under positive $\mathrm{g}$ the electrocardiogram shows certain changes which are mostly attributable to alterations in the electrical axis and pulse rate. The changes are never striking and in most subjects respiration caused greater changes than positive acceleration. Curiously enough, respiration under $\mathrm{g}$ caused as much change in the cardiogram as it did at rest, suggesting that abdominal breathing is still the most important means of pulmonary ventilation in spite of descent of the diaphragm.

In the instrumented runs here analysed the only cardiographic abnormality found was the occasional supraventricular extrasystole which, however, occurred unrelated to g. Some authors (Gauer, 1950; Zuidema et al., 1956) have commented on $T$ wave changes and concluded that they are indicative of myocardial anoxia. Reference to Fig. 4 and 5, however, shows that marked $\mathrm{T}$ wave changes occur during respiration and are positional in origin. In only one record when the subject lost consciousness was there even a hint of ventricular strain (Fig. 2). In view of the possibility of ventricular strain, it was thought that more subtle analysis of records might give 
evidence of minor degrees of strain when tolerance was being exceeded. The ventricular gradient, i.e. the relationship between excitation (QRS) and repolarization (RS-T and T), is said to be useful in interpreting the significance of $T$ wave changes (Houssay, 1955). In some preliminary calculations it was found that the ventricular gradient did in fact alter under $\mathrm{g}$, but, as the gradient varies both with position and pulse rate, the method was abandoned as an index of ventricular strain.

One of the striking findings in this investigation was the relatively small effect of positive acceleration on the electrical axis compared with the effect of respiration. However, this result is perhaps not so surprising, for at $5 \mathrm{~g}$ the diaphragmatic descent is only $6 \mathrm{~cm}$., a figure easily reached by respiration. Another factor may be the lack of correspondence between anatomical axis measured from X-ray films and electrical axis measured from the cardiogram.

In conclusion it may be stated that at the levels of acceleration used there is no characteristic change in the electrocardiogram.

\section{SUMMARY}

Electrocardiographic changes under positive accelerative forces have been studied in the human centrifuge.

During $g$ the pulse rate increases. Subjects who are not used to riding on the centrifuge show greater increases in rate at $3 \mathrm{~g}$ compared with experienced subjects. At higher $\mathrm{g}$ this difference disappears. The reasons for this are discussed.

No characteristic cardiographic abnormalities were observed and the evidence for ventricular strain is very slender.

Analysis of the electrical axis showed changes under $\mathrm{g}$, but these were little different in magnitude from the changes occurring during respiration. In three subjects no correlation between electrical and anatomical axes was found.

\section{REFERENCES}

British Cardiac Society (1949). Brit. Heart J., 11, 103.

Browne, M. K. (1957). Nature, 180, 192.

, and Fitzsimons, J. T. (1957). Unpublished data.

Euler, W. S. von, and Lundberg, W. (1954). J. App. Physiol., 6, 551.

Gauer, O. H. (1950). In German Aviation Medicine World War II, 1. Washington, Dept. of the Air Force, p. $570-576$.

Goldberger, E. (1953). Unipolar Lead Electrocardiography and Vectorcardiography. Henry Kimpton, London.

Houssay, B. (1955). Human Physiology. McGraw-Hill Publishing Co. Ltd., London.

Wilson, F. N., and Johnson, F. D., McLeod, A. G., and Barker, P. S. (1934). Amer. Heart J., 9, 477.

Zuidema, G. D., Cohen, S. I., Silverman, A. J., and Riley, M. B. (1956). J. Aviat. Med., $27,469$. 